\title{
Real-life experience on effectiveness and safety of dupilumab in adult patients with moderate-to- severe atopic dermatitis
}

MC Fargnoli, M Esposito, S Ferrucci, G Girolomoni, A Offidani, A Patrizi, K Peris, A Costanzo, G Malara, G Pellacani, M Romanelli, P Amerio, A Cristaudo, ML Flori, A Motolese, P Betto, C Patruno, P Pigatto, R Sirna, G Stinco, I Zalaudek, L Bianchi, V Boccaletti, SP Cannavò, F Cusano, S Lembo, R Mozzillo, R Gallo, C Potenza, F Rongioletti, R Tiberio, T Grieco, G Micali, S Persechino, M Pettinato, S Pucci, E Savi, L Stingeni, A Romano, G Argenziano \& On behalf of the Dupilumab Italian National Access Program (Dup-INAP group)

To cite this article: MC Fargnoli, M Esposito, S Ferrucci, G Girolomoni, A Offidani, A Patrizi, K Peris, A Costanzo, G Malara, G Pellacani, M Romanelli, P Amerio, A Cristaudo, ML Flori, A Motolese, P Betto, C Patruno, P Pigatto, R Sirna, G Stinco, I Zalaudek, L Bianchi, V Boccaletti, SP Cannavò, F Cusano, S Lembo, R Mozzillo, R Gallo, C Potenza, F Rongioletti, R Tiberio, T Grieco, G Micali, S Persechino, M Pettinato, S Pucci, E Savi, L Stingeni, A Romano, G Argenziano \& On behalf of the Dupilumab Italian National Access Program (Dup-INAP group) (2019): Reallife experience on effectiveness and safety of dupilumab in adult patients with moderate-to-severe atopic dermatitis, Journal of Dermatological Treatment, DOI: 10.1080/09546634.2019.1682503

To link to this article: https://doi.org/10.1080/09546634.2019.1682503

Accepted author version posted online: 24 Oct 2019.

View related articles ๘
Submit your article to this journal $\widetilde{ }$

View Crossmark data $\nearrow$ 


\section{REAL-LIFE EXPERIENCE ON EFFECTIVENESS AND SAFETY OF DUPILUMAB IN ADULT PATIENTS WITH MODERATE-TO-SEVERE ATOPIC DERMATITIS}

Running head: Real-life experience with Dupilumab

Fargnoli MC, ${ }^{1 *}$ Esposito $\mathrm{M},{ }^{1 *}$ Ferrucci $\mathrm{S},{ }^{2}$ Girolomoni G, ${ }^{3}$ Offidani A, ${ }^{4}$ Patrizi A, ${ }^{5}$ Peris K, ${ }^{6,7}$ Costanzo A,${ }^{8}$ Malara G, ${ }^{9}$ Pellacani G, ${ }^{10}$ Romanelli M, ${ }^{11}$ Amerio $\mathrm{P},{ }^{12}$ Cristaudo A, ${ }^{13}$ Flori ML, ${ }^{14}$ Motolese A,${ }^{15}$ Betto $\mathrm{P},{ }^{16}$ Patruno $\mathrm{C},{ }^{17}$ Pigatto $\mathrm{P},{ }^{18}$ Sirna $\mathrm{R},{ }^{19}$ Stinco $\mathrm{G},{ }^{20}$ Zalaudek I, ${ }^{21}$ Bianchi $\mathrm{L},{ }^{22}$ Boccaletti V, ${ }^{23}$ Cannavò SP, ${ }^{24}$ Cusano $F,{ }^{25}$ Lembo $S,{ }^{26}$ Mozzillo $\mathrm{R},{ }^{27}$ Gallo $\mathrm{R},{ }^{28}$ Potenza $\mathrm{C},{ }^{29}$ Rongioletti F, ${ }^{30}$ Tiberio $\mathrm{R},{ }^{31}$ Grieco $\mathrm{T},{ }^{32}$ Micali G, ${ }^{33}$ Persechino $\mathrm{S},{ }^{34}$ Pettinato $\mathrm{M},{ }^{35}$ Pucci $\mathrm{S},{ }^{36}$ Savi E ${ }^{37}$ Stingeni $\mathrm{L},{ }^{38}$ Romano $\mathrm{A},{ }^{39}$ Argenziano $\mathrm{G}^{40}$. On behalf of the Dupilumab Italian National Access Program (Dup-INAP group).

*equally contributed

${ }^{1}$ Department of Dermatology, University of L'Aquila; ${ }^{2}$ Unit of Dermatology, Fondazione IRCCS Ca' Grande Ospedale Maggiore Policlinico, Milan; ${ }^{3}$ Section of Dermatology and Venereology, Department of Medicine, University of Verona; ${ }^{4}$ Department of Clinical and Molecular Sciences, Dermatology Unit, Polytechnic Marche University, Ancona; ${ }^{5}$ Dermatology Unit, Department of Experimental, Diagnostic and Specialty Medicine, University of Bologna; ${ }^{6}$ Institute of Dermatology, Catholic University, Rome; ${ }^{7}$ Fondazione Policlinico Universitario A. Gemelli IRCCS, Rome; ${ }^{8}$ Dermatology Unit, Department of Biomedical Sciences, Humanitas University and Humanitas Clinical and Research Center, Rozzano; ${ }^{9}$ Dermatology Department, Grande Ospedale Metropolitano, Bianchi Melacrino Morelli, Reggio Calabria, ${ }^{10}$ Department of Dermatology, University of Modena and Reggio Emilia, Modena; ${ }^{11}$ Dermatology Unit, Department of Clinical and Experimental Medicine, University of Pisa; ${ }^{12}$ Department of Medicine and Aging Science, Dermatologic Clinic, G. D'Annunzio University, Chieti; ${ }^{13}$ Unit of Dermatology, San Gallicano Dermatological Institute-IRCCS, Rome; ${ }^{14}$ Dermatology Section, Department of Clinical Medicine and Immunological Science, University of Siena, Hospital S. Maria alle Scotte, Siena; ${ }^{15}$ Department of Dermatology, Macchi Hospital, Varese; ${ }^{16}$ Department of Dermatology, Ospedale San Bortolo, ULSS8 Berica, Vicenza; ${ }^{17}$ Department of Health Sciences, University Magna Graecia of Catanzaro, Catanzaro; ${ }^{18}$ Department of Biomedical, Surgical and Dental 
Sciences, Clinical Dermatology, IRCCS Galeazzi Orthopaedic Institute, University of Milan;

${ }^{19}$ Unit of Dermatology, Ospedale della Misericordia, Grosseto; ${ }^{20}$ Department of Medicine, Institute of Dermatology, University of Udine; ${ }^{21}$ Dermatology Clinic, Hospital Maggiore of Trieste; ${ }^{22}$ Department of Dermatology, University of Tor Vergata, Rome; ${ }^{23}$ Department of Clinical and Experimental Medicine, Section of Dermatology, University Hospital, Parma; ${ }^{24}$ Department of Clinical and Experimental Medicine, Dermatology, University of Messina; ${ }^{25}$ Dermatology Unit, Gaetano Rummo Hospital Benevento; ${ }^{26}$ Unit of Dermatology, University Hospital San Giovanni di Dio e Ruggi di Salerno, Hospital Santa Maria Incoronata dell'Olmo, Cava de' Tirreni, Salerno; ${ }^{27}$ Dermatology and Venereology, "San Gennaro" Hospital, Naples; ${ }^{28}$ Dermatology Section, Dept of Health Science, University of Genoa, Polyclinic Hospital San Martino, Genoa; ${ }^{29}$ Department of Medical and Surgical Sciences and Biotechnologies, Dermatology Unit Daniele Innocenzi, Sapienza University of Rome - Polo Pontino; ${ }^{30}$ Department of Medical Sciences and Public Health, Section of Dermatology, University of Cagliari; ${ }^{31}$ Department of Health Science, University of Eastern Piedmont, Novara; ${ }^{32}$ Dermatology Clinic Umberto I, Sapienza University of Rome; ${ }^{33}$ Dermatology Clinic, University of Catania, Catania, Italy; ${ }^{34}$ Dermatology Unit, Sant'Andrea Hospital, NESMOS Department, Sapienza University of Rome; ${ }^{35}$ Unit of Dermatology, University Hospital, Policlinico Vittorio Emanuele, Catania; ${ }^{36}$ Allergology, Hospital of Civitanova Marche; ${ }^{37}$ Unit of Dermatology, Hospital Guglielmo da Saliceto, Piacenza; ${ }^{38}$ Dermatology Section, Department of Medicine, University of Perugia; ${ }^{39}$ Allergology Unit, Columbus Presidium, Catholic University, Fondazione Policlinico Universitario A. Gemelli IRCCS, Rome; ${ }^{40}$ Dermatology Unit, University of Campania Luigi Vanvitelli, Naples.

Members of the Dup-INAP GROUP:

Giulia Calabrese, Camilla Chello, Tiziana De Pasquale, Luisa Di Costanzo, Valentina Dini, Rocco De Pasquale, Micol Del Giglio, Giovanna Fracassi, Niccolò Gori, Domenico Giordano, Alessandro Giunta, Carlotta Gurioli, Katharina Hansel, Agostina Legori, Camilla Loi, Giuseppe Matteo Maini, Ilenia Marafioti, Giovanna Meogrossi, Katiuscia Nan, Maddalena Napolitano, Diego Orsini, Maria Parodi, Camilla Peccianti, Cristina Pellegrini, Viviana Piras, Annunziata Raimondo, Filomena Russo, Marina Talamonti, Simona Tavecchio, Caterina Trifirò, Mario Valenti, Federica Veronese, Martina Vispi, Yiran Wei. 
KEY WORDS: atopic dermatitis, dupilumab, real-life

Word count: 2133

\section{Number of references: 21}

Number of figures: 3

Number of tables: 1

Correspondence: Maria Esposito, MD, Department of Dermatology, University of L'Aquila, via Vetoio-Coppito 2, 67100 L'Aquila, Italy; Tel: +39-0862-368519; Fax: +39-0862-433433; e-mail: espositomaria@virgilio.it

\section{Conflicts of interest:}

P. Amerio received honoraria as a speaker and advisory board member from SanofiGenzyme. P. Betto has been principal investigator in clinical trials sponsored by and/or received honoraria from Sanofi-Genzyme and Sandoz. L. Bianchi has been principal investigator in clinical trials sponsored by and/or received personal fees from Sanofi Genzyme, Eli-Lilly, Abbvie. S.P. Cannavò has been principal investigator in clinical trials sponsored by and/or received personal fees from AbbVie, Celgene, Eli-Lilly, Janssen-Cilag, Leo Pharma, Novartis, Sanofi. A. Costanzo has been principal investigator in clinical trials sponsored by and/or received personal fees from AbbVie, Abiogen, Almirall, Amgen, Biogen, Celgene, Eli-Lilly, Genzyme, Leo Pharma, Novartis, Pfizer, Regeneron, Samsung, Sandoz and Sanofi. A. Cristaudo has been principal investigator in clinical trials sponsored by Abbvie, Leo Pharma and Pfizer. F. Cusano received honoraria as speaker and advisory board member from Sanofi Genzyme. M. Esposito has served as a speaker for Sanofi-Genzyme. M.C. Fargnoli has served on advisory board, received honoraria for lectures and research grants from Sanofi-Genzyme. S. Ferrucci has been principal investigator in clinical trials sponsored by and/or received personal fees from AbbVie, Eli-Lilly, Novartis, and Sanofi. G. Girolomoni has been principal investigator in clinical trials sponsored by and/or received personal fees from AbbVie, Abiogen, Almirall, Amgen, Biogen, Celgene, Eli-Lilly, Genzyme, Leo Pharma, Menlo therapeutics, Novartis, Pfizer, Regeneron, Samsung, Sandoz and Sanofi. S. Lembo has received honoraria as a consultant and for attending an academic international meeting. G. Malara has been principal investigator in clinical trials sponsored by 
and/or received personal fees from AbbVie, Eli-Lilly, Janssen, Celgene, Sanofi and Almirall. A. Offidani has been principal investigator in clinical trials and has been paid as consultant by AbbVie, Almirall, Amgen, Celgene, Eli-Lilly, Leo Pharma, Novartis, Pfizer, Regeneroni and Sanofi. A Patrizi has served as a speaker and received honoraria from Sanofi-Genzyme for lectures, research grants and as an advisory board member. C. Patruno has been reimbursed by Sanofi for Advisory Boards and for international conference attendance. G. Pellacani has been principal investigator in clinical trials sponsored by and/or received personal fees from AbbVie, Almirall, Eli-Lilly, Leo Pharma, Novartis, and Sanofi. K. Peris has received honoraria as speaker and advisory board member from Sanofi-Genzyme. F. Rongioletti has served on advisory board, received honoraria for lectures and research grants from Nôvartis, AbbVie, Janssen-Cilag, Eli-Lilly, Leo Pharma, Sanofi-Genzyme. G. Stinco has been principal investigator in clinical trials, received honoraria for lectures and research grants from Novartis, AbbVie, Janssen-Cilag, Eli-Lilly, Leo Pharma, Sandoz, UCB. L. Stingeni has been principal investigator in clinical trials sponsored by and/or received personal fees from AbbVie, Almirall, Celgene, Eli-Lilly, Janssen, Novartis, and Sanofi-Genzyme. R. Tiberio has been principal investigator in clinical trials and received honoraria by Sanofi-Genzyme, Abbvie, Eli-Lilly.

Funding sources: This research was not funded. 


\section{ABSTRACT}

Background: Dupilumab, a fully human monoclonal antibody targeting the alpha subunit of IL-4 was recently approved for the treatment of moderate-to-severe atopic dermatitis (AD) in adult patients.

Objective: To assess dupilumab effectiveness and safety in adults with moderate-to-severe $\mathrm{AD}$ in a real-life Italian multicentre retrospective cohort.

Methods: Adult moderate-to-severe AD patients, referring to 39 Italian centres, received dupilumab in the context of a national patient access program. Disease assessment was performed at baseline, after 4 and 16 weeks of treatment using Eczema-Area-and-SeverityIndex (EASI) score, itch and sleep numerical-rating-score (itch-NRS, sleep-NRS) and Dermatology-Life-Quality-Index (DLQI).

Results: A total of $109(71 \mathrm{M} / 38 \mathrm{~F})$ patients was studied. There was a significant reduction in EASI score, itch-NRS, sleep-NRS and DLQI from baseline to week 4 and a further significant decline to week 16. EASI 50, EASI75 and EASI90 were achieved by 59.6\%, 28.4\% and 9.3\% of patients at 4 weeks and by $87.2 \%, 60.6 \%$ and $32.4 \%$ of them at 16 weeks, respectively. Adverse events were experienced by $19.2 \%$ (21/109) of the patients and they were all mild in intensity, being conjunctivitis the most common side effect.

Conclusions: Dupilumab significantly improved disease severity, pruritus, sleep loss and quality of life with an acceptable safety profile. 


\section{INTRODUCTION}

Atopic dermatitis (AD) is a chronic inflammatory skin disease affecting 7-10\% of the adult population [1]. The disease is characterized by pruritic eczematous lesions with a high heterogeneity of clinical manifestations [2]. Approximately $10 \%$ of adults with AD do not respond adequately to topical therapies and require phototherapy and/or systemic therapy [3]. Cyclosporine has been the only immunosuppressive drug approved for the treatment of AD in many countries so far, but its use is limited by potential long-term organ toxicity and ineffectiveness [4]. Second-line immunosuppressants, such as azathioprine, methotrexate and mycophenolate mofetil, represent off-label options suggested by international guidelines, but data on their long-term effectiveness and safety are lacking [5].

Th2-related cytokines, interleukin-4 (IL-4) and IL-13, play a key role in AD pathophysiology. Dupilumab is a fully human monoclonal antibody targeting the alpha subunit of IL-4 (IL-4R $\alpha$ ), common to IL-4 and IL-13, thus blocking signaling of both cytokines [6]. It is the first targeted biologic agent approved by FDA and EMA for the treatment of moderate-to-severe $\mathrm{AD}$ in adults. Randomized clinical trials demonstrated efficacy of dupilumab on objective and subjective disease parameters and on quality of life [7-9]. However, studies on real-life experience are needed to address dupilumab treatment since patients included in clinical trials represent a selected population, not reflecting clinical practice.

Aim of the present study was the retrospective assessment of dupilumab effectiveness and safety in a real-life Italian multicentre cohort of adult moderate-to-severe $\mathrm{AD}$ patients. 


\section{METHODS}

We retrospectively collected data of adult patients with moderate-to-severe AD treated with dupilumab from June 2018 to February 2019 at 39 dermatological referral centres in the context of a national patient access program authorized by the Italian Medical Agency. Treatment eligibility criteria were age $\geq 18$ years, diagnosis of moderate-to-severe AD [10], Eczema-Area-and-Severity-Index [11] (EASI) $\geq 24$, contraindication, side effects or failure in respect to cyclosporine (all established criteria for patient enrollment in the national access program by the Italian Medical Agency). No specific wash-out period was required. Patients were consecutively included in the analysis if they achieved an observation period of at least 16 weeks. Exclusion criteria were concomitant use of systemic immunomodulating agents and non-availability/incompleteness of recorded patients' data. Patients were treated with subcutaneous dupilumab at an initial loading dose of $600 \mathrm{mg}$ followed by $300 \mathrm{mg}$ every other week (label use). The following data were collected from medical records: demographic variables, disease pattern, AD phenotype, special locations of lesions (face, hands, genitals), comorbidities, previous treatments, changes of objective and subjective disease parameters and adverse events. Eosinophil count and total serum immunoglobulin E (IgE) levels were also collected, when available. Disease severity was assessed at baseline, after 4 and 16 weeks of treatment using EASI score (range 0-72) [11], itch and sleep numerical-rating-score (itchNRS and sleep-NRS) (range 0-10) evaluated as peak score during the past 24 hours $[12,13]$ and Dermatology Life Quality Index (DLQI) score (range 0-30) [14]. Patients' evaluation was performed at week 4 and 16 with a window of assessment of +/- 2 days due to timing of drug distribution.

A signed informed consent was obtained from patients allowing to extract data from clinical records. Approval of the study protocol was obtained by the Internal Review Board of the University of L'Aquila (no. 27/2019). 


\section{Statistical analysis}

All statistical analysis was conducted with last observation carried forward population to account for missing data. Descriptive statistics are given as mean, medians or proportion. Categorical variables were classified into two groups, above or below the median. Eosinophilia was defined as $>500$ cells $/ \mathrm{mm}^{3}$. Total IgE normal values were considered $\operatorname{IgE}<$ $100 \mathrm{kU} / \mathrm{L}$. The effectiveness of treatment was evaluated at week 4 and at week 16 in terms of mean percentage change from baseline and percentage of patients with an EASI reduction $\geq 50 \%, \geq 75 \%$ and $\geq 90 \%$ from baseline (EASI50, EASI75, EASI 90 ).

Wilcoxon matched-pairs rank or paired $\mathrm{T}$ tests were used to evaluate differences between baseline and follow-ups. The relationship between IgE and EASI score was analyzed by Spearman's correlation. Fisher's exact test was used to test the association among EASI75, age, sex, body mass index (BMI), duration of disease, baseline EASI score. Statistics were performed by IBM-SPSS softwarev.25 (Chicago, USA). Differences were significant if $\mathrm{p}<$ 0.05 .

\section{RESULTS}

Study population

A total of $109(71 \mathrm{M} / 38 \mathrm{~F})$ patients, with a mean age of 37.9 years (SD 14.7, range 1980) was included in the study. Demographic and clinical baseline characteristics of patients are summarized in Table 1. Mean age at disease onset was 14.2 years (range 0-77, SD 17.8), mean BMI was 23.9 (range 17-34.6, SD 3.4) and the pattern of AD was persistent in 59/109 (54.1\%) patients, relapsing in 27/109 (24.8\%) patients and late-onset in 23/109 (21.1\%). The most frequent $\mathrm{AD}$ phenotype was the classic adult-type with lichenified/exudative flexural 
dermatitis, often associated with head/neck eczema and/or hand/feet eczema, observed in $79 / 109(72.5 \%)$ patients, followed by erythrodermic in 13/109 (13\%), prurigo in $9 / 109(8.2 \%)$ and nummular dermatitis in 8/109 (7.3\%). Face was affected in 83/109 (76.1\%) patients, hands in 67/109 (61.5\%) and genitals in 25/109 (22.9\%). Allergic comorbidities were mostly represented by allergic rhinitis (44.9\%), asthma (38.5\%), conjunctivitis (33.0\%) and food allergy (15.6\%). Other comorbidities including psychiatric or psychological conditions (11\%), hypertension and cardiovascular disorders (9.1\%) and obesity (6.4\%) were less frequent. The majority of patients had been treated with cyclosporine $(88.9 \%)$, followed by oral corticosteroids (88.1\%), phototherapy (UVB) $(45.8 \%)$, methotrexate $(24.7 \%)$, azathioprine (16.5\%), omalizumab (7.3\%) and mycophenolate mofetil (0.9\%).

\section{Effectiveness parameters}

A total of 106/109 patients completed the 16-week treatment period. Three patients discontinued treatment at week 4, due to ano-rectal bleeding for an anal ragad (1), inefficacy (1) and patient choice (1).

There was a significant reduction in EASI score, itch-NRS, sleep-NRS and DLQI score between baseline to 4 weeks and a further significant decline to 16 weeks. Mean variation of EASI (A), itch-NRS (B), sleep-NRS (C) and DLQI (D) from baseline at both week 4 and week 16 are shown in Figure 1 and EASI50, EASI75 and EASI90 results in Figure 2.

At baseline, the mean EASI score was 33.3 (SD 16.1) and was significantly reduced to 15.3 (SD 12.7) at 4 weeks and to 9.2 (SD 10.2) at 16 weeks ( $<<0.001$ for all comparisons). Mean EASI score percentage reduction was $54.2 \%$ at 4 weeks and $72.5 \%$ at 16 weeks. At 4 weeks, $59.6 \%$ of patients achieved EASI50, and this percentage increased to $87.2 \%$ at 16 
weeks. EASI75 and EASI90 were achieved by $28.4 \%$ and $9.3 \%$ of patients at 4 weeks and by $60.6 \%$ and $32.4 \%$ of them at 16 weeks, respectively.

Itch-NRS had a mean value of 8.4 (SD 1.3) at baseline, 4.1 (SD 2.3) at 4 weeks and 2.5 (SD 2.6) at 16 weeks ( $<<0.001$ for all comparisons). Mean itch-NRS percentage reduction was $51.5 \%$ at week 4 and $69.5 \%$ at week 16 .

The mean baseline values of sleep-NRS significantly reduced from 6.9 (SD 2.5) to 3.3 (SD 2.4) at 4 weeks and to 1.9 (SD 2.2) at 16 weeks ( $<<0.001$ for all comparisons). Mean sleep-NRS percentage reduction was $52.5 \%$ at 4 weeks and $72.3 \%$ at 16 weeks.

As for the quality of life index, mean DLQI score was 17.6 (SD 6.2) at baseline, 8.3 (SD 6.4) at 4 weeks and 5.4 (SD 6.2) at 16 weeks ( $<<0.001$ for all comparison). Mean DLQI score percentage reduction was $52.9 \%$ at 4 weeks and $69.1 \%$ at week 16 .

Sex, age, BMI, disease duration and baseline EASI were not significantly associated to EASI75 at 4 weeks ( $\mathrm{p}=0.279 ; \mathrm{p}=0.832 ; \mathrm{p}=0.880, \mathrm{p}=0.606, \mathrm{p}=0.140$, respectively) and at 16 weeks ( $\mathrm{p}=0.833 ; \mathrm{p}=0.538 ; \mathrm{p}=0.211, \mathrm{p}=0.065, \mathrm{p}=0.420$, respectively).

Eosinophilia $\left(>500 / \mathrm{mm}^{3}\right)$ was detected in $36.7 \%$ (40/109) of patients at baseline, in $42.2 \%$ (46/109 patients) at week 4 and in $58.7 \%$ (64/109 patients) at week 16.

Total IgE serum levels were available in 66/109 (60.5\%) patients both at baseline and at the 16-week follow-up visit. At baseline, total IgE levels were above normal in 53/66 $(80.3 \%)$ patients with a mean value of $5099 \times 10^{3}$ IU/L (SD 7728) that decreased in 40/53 (75\%) patients to a mean value of $4059 \times 10^{3} \mathrm{IU} / \mathrm{L}$ (SD 6708) at week 16. IgE levels were stable during treatment period in the remaining 13/53 (25\%) patients.

At baseline, a positive correlation was found between total serum $\operatorname{IgE}$ and baseline EASI score $(\mathrm{r}=0.33, \mathrm{p}=0.002)$. Moreover, significant direct correlations were observed between baseline $\operatorname{IgE}$ and relative reduction of EASI score at 4 weeks $(r=0.26, p=0.021)$ and 
at 16 weeks $(\mathrm{p}<0.001)$.

\section{Safety profile}

At least one adverse event was experienced by $19.2 \%$ (21/109) of the patients and they were all mild in intensity. Conjunctivitis was the most common side effect being observed in 12/109 patients (11\%), followed by arthralgia (2), headache (2), injection site reaction (2), fatigue (1), folliculitis (1) and ano-rectal bleeding for an anal ragad leading to treatment interruption (1). In our patients, conjunctivitis was mostly treated with artificial tears or hyaluronic acid eye-drops and resolved spontaneously. A pharmacologic topical approach with steroids, antimicrobials or cyclosporine, was required in a minority of the cases.

\section{DISCUSSION}

Our results confirmed the effectiveness and safety of dupilumab in a real-life setting in adult moderate-to-severe AD patients, as expressed by a significant reduction of all disease objective (EASI) (Figure 3) and subjective (itch-NRS, sleep-NRS) scores and of quality of life (DLQI) index as well as total IgE serum levels during the study period. Improvement in all effectiveness and quality of life parameters was significant between baseline and week 4 and between week 4 and week 16 .

Few European real-life experiences have been reported in France, Denmark, Spain and The Netherlands [15-20]. In a small single-center observational study, dupilumab was proved to improve AD manifestations in 19 patients, in terms of global severity, pruritus, sleep loss and effect on atopic comorbidities [15]. At week 16, median SCORAD decreased by 55\% and SCORAD75 was achieved by $22 \%$ of patients. A larger multicenter French study in 241 adult 
AD patients reported EASI75 in $48.8 \%$ of the patients after 3 months. A high frequency of conjunctivitis $(38.2 \%)$ and eosinophilia $(56.5 \%)$ with hypereosinophilia in one third of the patients and a high discontinuation rate (17.4\%) characterized this patients' cohort [16]. In 43 Danish $\mathrm{AD}$ patients, signs and symptoms of $\mathrm{AD}$ improved after 3 months of dupilumab treatment with a statistically significant reduction in EASI, DLQI, pruritus and sleep VAS scores, serum IgE and LDH [17]. The overall percentage improvement of EASI was $72.4 \%$ at 1 month and $76.7 \%$ at 3 months. Main results were observed within the first month of treatment with continuous but not significant further improvement between one and three months. A Spanish real-life case series of $70 \mathrm{AD}$ patients demonstrated a $79.3 \%$ EASI reduction and a decrease of pruritus VAS of $69.9 \%$ and of DLQI value of $62.8 \%$ at week 24 [18]. Long-term maintenance of response with no emerging adverse event was demonstrated in a small series of 30 Spanish patients treated with dupilumab for 52 weeks [19]. Finally, an observational study in The Netherlands described 95 patients treated with dupilumab with concomitant conventional systemic immunosuppressants in 43 patients, demonstrating that the combination regimen is an effective and safe transition to monotherapy [20].

In our real-life cohort, a $72.5 \%$ percentage reduction of the mean EASI score was achieved from baseline to week 16 with $87.2 \%, 60.6 \%$ and $32.4 \%$ of patients reaching EASI50, EASI75 and EASI90, respectively. The percentage of patients with EASI50 and EASI75 at week 16 was similar to that observed in clinical trials investigating dupilumab in combination with topical corticosteroids [8,9] while it was higher than in trials using dupilumab as monotherapy [7]. Concomitant treatment with topical anti-inflammatory agents is common practice in the real-life dermatological setting and our results are indeed in line with other real-life studies [17].

Dupilumab markedly improved key symptoms in AD positively influencing patient's quality of life by significantly reducing mean itch-NRS from baseline 8.4 to 2.5 at 16 weeks 
and mean sleep-NRS from baseline 6.9 to 1.9 at 16 weeks with a mean DLQI score percentage reduction of $69.1 \%$ at week 16 .

In line with previous studies, the improvement in signs and symptoms was accompanied with a decrease of total serum $\operatorname{IgE}[15,17]$. This finding is expected due to the specific mechanism of action of dupilumab blocking IL-4 and IL-13 known to increase IgE production. In addition, we found a positive correlation between total serum $\operatorname{IgE}$ and baseline EASI score and between baseline IgE and reduction of EASI score at weeks 4 and 16.

Dupilumab safety profile was favorable. Conjunctivitis, clinically confirmed by an oftalmologist, was the most frequent adverse event, observed in $11 \%$ of our patients, always mild in intensity and successfully resolved with topical therapy. History of allergic conjunctivitis was present in $9 / 12$ cases $(75 \%)$. Conjunctivitis was reported in $8.6-22.1 \%$ of AD patients in 11 dupilumab trials [21] and ranges up to $40 \%$ in real-life studies [16-19]. Management of conjunctivitis requires collaboration with experienced ophtalmologists and a graded therapeutic approach [21].

Limitations of our study include the small size of the cohort, the short follow-up period and the retrospective design of the study. On the other hand, real-world experiences of new treatments are needed since they have been only used in clinical trials in selected patients.

In conclusion, dupilumab demonstrated effectiveness in reducing disease severity, pruritus, sleep loss and improved quality of life with a favorable safety profile in our series of adult patients with moderate-to-severe, difficult-to-treat $\mathrm{AD}$, confirming data of clinical trials. Further studies are needed to investigate the patterns of treatment response according to the heterogeneous $\mathrm{AD}$ phenotypes and the long-term effectiveness and safety of the drug. 


\section{REFERENCES}

1. Weidinger S, Beck LA, Bieber T et al. Atopic dermatitis. Nature reviews Dis Primers $2018 ; 21 ; 4: 1$.

2. Megna M, Patruno C, Balato A et al. An Italian multicentre study on adult atopic dermatitis: persistent versus adult-onset disease. Arch Dermatol Res 2017;309:443452.

3. Ariëns LFM, Bakker DS, van der Schaft $\mathbf{J}$ et al. Dupilumab in atopic dermatitis: rationale, latest evidence and place in therapy. Ther Adv Chronic Dis 2018;9:159-170.

4. van der Schaft J, Politiek K, van den Reek JM, et al. Drug survival for ciclosporin A in a long-term daily practice cohort of adult patients with atopic dermatitis. $\mathrm{Br} J$ Dermatol 2015;17:1621-1627.

5. Wollenberg A, Barbarot S, Bieber T et al. European Dermatology Forum (EDF), the European Academy of Dermatology and Venereology (EADV), European Academy of Allergy and Clinical Immunology (EAACI), European Task Force on Atopic Dermatitis (ETFAD), European Federation of Allergy and Airways Diseases Patients' Associations (EFA), European Society for Dermatology and Psychiatry (ESDaP), European Society of Pediatric Dermatology (ESPD), Global Allergy and Asthma European Network (GA2LEN) and European Union of Medical Specialists (UEMS). Consensus-based European guidelines for treatment of atopic eczema (atopic dermatitis) in adults and children: part II. J Eur Acad Dermatol Venereol 2018;32:850-878.

6. Beck LA, Thaçi D, Hamilton JD, et al. Dupilumab treatment in adults with moderateto-severe atopic dermatitis. N Engl J Med 2014;371:130-139.

7. Simpson EL, Bieber T, Guttman-Yassky E et al. Two Phase 3 Trials of Dupilumab 
versus Placebo in Atopic Dermatitis. N Engl J Med 2016;375:2335-2348.

8. Blauvelt A, de Bruin-Weller M, Gooderham M et al. Long-term management of moderate-to-severe atopic dermatitis with dupilumab and concomitant topical corticosteroids (LIBERTY AD CHRONOS): a 1-year, randomised, double-blinded, placebo-controlled, phase 3 trial. Lancet 2017;389:2287-2303.

9. de Bruin-Weller M, Thaçi D, Smith $\mathrm{CH}$ et al. Dupilumab with concomitant topical corticosteroids in adult patients with atopic dermatitis who are not adequately controlled with or are intolerant to ciclosporin A, or when this treatment is medically inadvisable: a placebo-controlled, randomized phase. Br J Dermatol 2018;178:10831101.

10. Hanifin JM, Rajka G. Diagnostic features of atopic dermatitis. Acta Derm Venereol Suppl (Stockholm) 1980;6:44-47.

11. Hanifin JM, Thurston M, Omoto M, et al. The eczema area and severity index (EASI): assessment of reliability in atopic dermatitis. EASI Evaluator Group. Exp Dermatol 2001;10:11-18.

12. Yosipovitch G, ReaneyM, Mastey V et al. Peak Pruritus Numerical Rating Scale: Psychometric Validation and Responder. Definition for Assessing Itch in ModerateTo-Severe Atopic Dermatitis. Br J Dermatol 2019 Feb 6. doi:10.1111/bjd.17744.

13. Silverberg JI, Chiesa Fuxench ZC, Gelfand JM et al. Content and construct validity, predictors, and distribution of self-reported atopic dermatitis severity in US adults. Ann Allergy Asthma Immunol 2018;121:729-734.

14. Badia X, Mascaró JM, Lozano R. Measuring health-related quality of life in patients with mild to moderate eczema and psoriasis: clinical validity, reliability and sensitivity to change of the DLQI. Br J Dermatol 1999;141:698-702. 
15. Tauber M, Apoil PA, Richet $\mathrm{C}$ et al. Effect of dupilumab on atopic manifestations in patients treated for atopic dermatitis in real-life practice. Br J Dermatol 2019 Jan 11. doi:10.1111/bjd.17629.

16. Faiz S, Giovannelli J, Podevin C et al. Effectiveness and safety of dupilumab for the treatment of atopic dermatitis in a real-life French multicenter adult cohort. J Am Acad Dermatol 2019 Feb 27. doi.org/10.1016/j.jaad.2019.02.053.

17. Olesen CM, Holm JG, Nørreslet LB et al. Treatment of atopic dermatitis with dupilumab: experience from a tertiary referral center. J Eur Acad Dermatol Venereol 2019 Apr 8. doi:10.1111/jdv.15609.

18. Armario-Hita JC, Pereyra-Rodriguez J, Silvestre JF et al. Treatment of moderate-to severe atopic dermatitis with dupilumab in real clinical practice. A multicentre, retrospective case series. Br J Dermatol 2019 Apr 25. doi:10.1111/bjd.18041.

19. Ruiz-Villaverde R, Dominguez-Cruz J, Armario-Hita JC et al. Fifty-two week followup safety and effectiveness results of dupilumab treatment of moderate-to-severe atopic dermatitis from a retrospective, multicentric series. Dermatol Ther 2019; 13:e129.

20. de Wijs LEM, Bosma AL, Erler NS et al. Effectiveness of dupilumab treatment in 95 patients with atopic dermatitis: daily practice data. Br J Dermatol 2019 May 30. doi: 10.1111/bjd.18179.

21. Akinlade B, Guttman-Yassky E, de Bruin-Weller $M$, et al. Conjunctivitis in dupilumab clinical trials. Br J Dermatol 2019 Mar 9. doi: 10.1111/bjd.17869. 


\section{LEGENDS}

Table 1. Demographic and clinical baseline patients' characteristics (109 patients)

\begin{tabular}{|c|c|}
\hline Gender & $\mathrm{n}(\%)$ \\
\hline Men & $71(65.1)$ \\
\hline Women & $38(34.9)$ \\
\hline Age & yrs \\
\hline Mean (SD) & $37.9(14.7)$ \\
\hline Range & $19-80$ \\
\hline Age at onset & yrs \\
\hline Mean (SD) & $14.2(17.8)$ \\
\hline Range & $0-77$ \\
\hline \multicolumn{2}{|l|}{ Body Mass Index } \\
\hline Mean (SD) & $23.9(3.4)$ \\
\hline Range & $17-34.6$ \\
\hline \multicolumn{2}{|l|}{ AD pattern } \\
\hline Persistent & $59(54.1)$ \\
\hline Relapsing & $27(24.8)$ \\
\hline Late-onset & $23(21.1)$ \\
\hline \multicolumn{2}{|l|}{ AD phenotype } \\
\hline Classic adult-type & $79(72.5)$ \\
\hline Erythrodermic $\mathrm{AD}$ & $13(12.0)$ \\
\hline Prurigo & $9(8.2)$ \\
\hline Nummular dermatitis & $8(7.3)$ \\
\hline Allergic comorbidities & n $(\%)$ \\
\hline Rhinitis & $49(44.9)$ \\
\hline Asthma & $42(38.5)$ \\
\hline Conjunctivitis & $36(33.0)$ \\
\hline Food Allergy & $17(15.6)$ \\
\hline Other Comorbidities & n (\%) \\
\hline Psychiatric/psychological conditions & $12(11.0)$ \\
\hline Hypertension and other cardioyascular disorders & $10(9.1)$ \\
\hline Obesity & $7(6.4)$ \\
\hline Neurological disorders & $6(5.5)$ \\
\hline Dyslipidaemia $\square$ & $5(4.6)$ \\
\hline Previous neoplastic disease & $4(3.6)$ \\
\hline Thyroid disorders & $4(3.6)$ \\
\hline Psoriasis & $3(2.7)$ \\
\hline Coeliac disease & $2(1.8)$ \\
\hline Diabetes & $1(0.9)$ \\
\hline Previous treatments & $\mathrm{n}(\%)$ \\
\hline Cyclosporine & $97(88.9)$ \\
\hline Oral Corticosteroids & $96(88.1)$ \\
\hline Phototherapy (UVB) & $50(45.8)$ \\
\hline Methotrexate & $27(24.7)$ \\
\hline Azathioprine & $18(16.5)$ \\
\hline
\end{tabular}




\begin{tabular}{|l|c|}
\hline \multicolumn{1}{|c|}{ Omalizumab } & $8(7.3)$ \\
\hline Mycophenolate mofetil & $1(0.9)$ \\
\hline Baseline mean EASI, value (SD) & $33.3(16.1)$ \\
\hline Baseline mean itch-NRS, value (SD) & $8.3(1.3)$ \\
\hline Baseline mean sleep-NRS, value (SD) & $6.9(2.5)$ \\
\hline Baseline mean DLQI, value (SD) & $17.6(6.2)$ \\
\hline
\end{tabular}

EASI, Eczema Area and Severity Index; NRS, numerical rating score; DLQI, Dermatology Life Quality Index

A

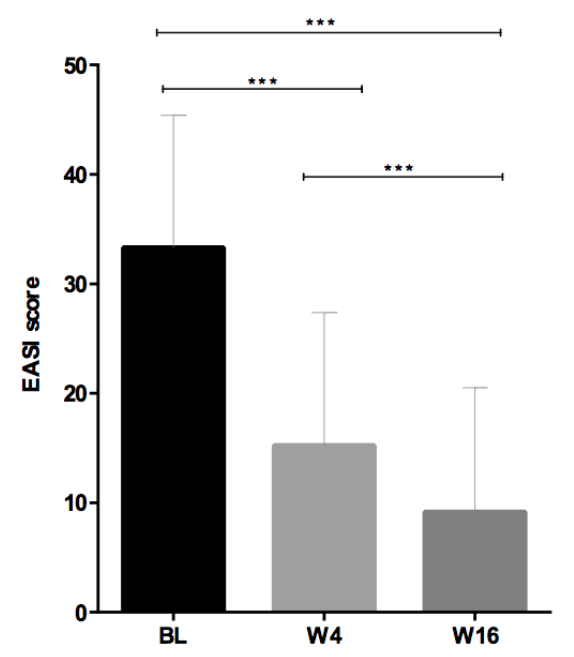

C

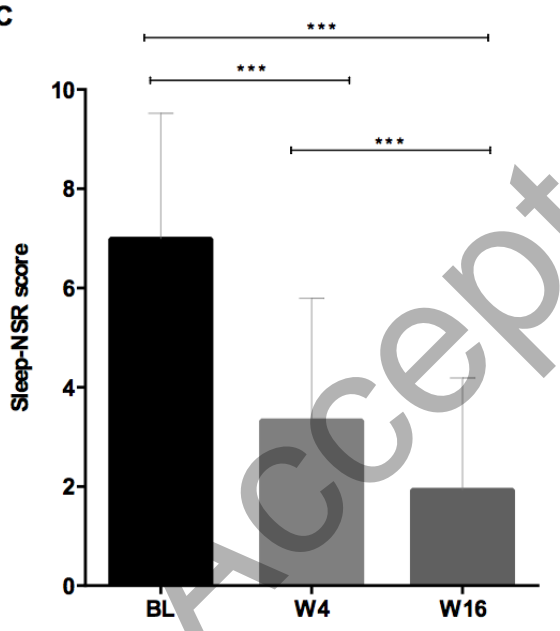

B

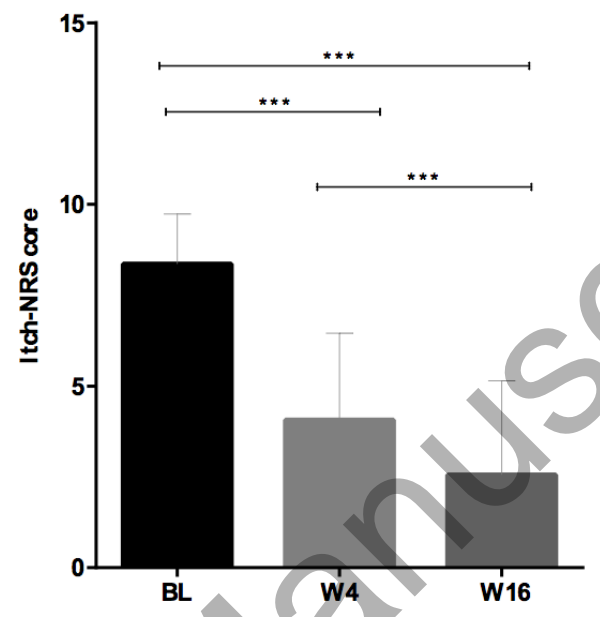

D

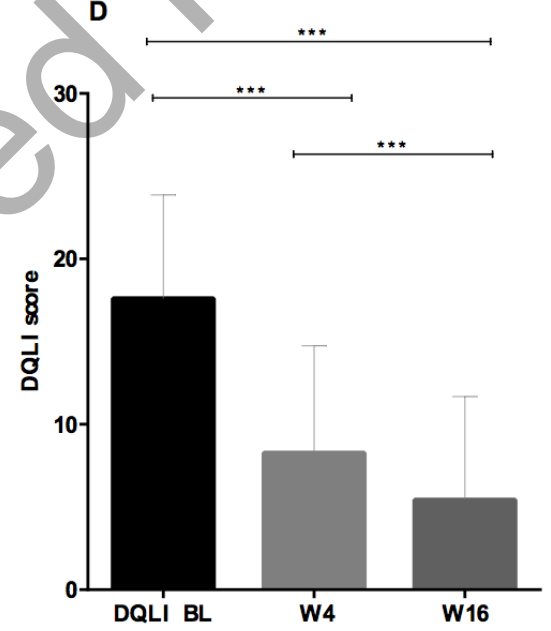

Figure 1. Results describing improvement in terms of mean variation of EASI (A), itch-NRS (B), sleep NRS (C) and DLQI (D) at week 4 and week 16. ***p $<0.0001$ for all comparisons. BL, baseline; W4, week 4; W16, week 16. Thin lines refer to SD. 


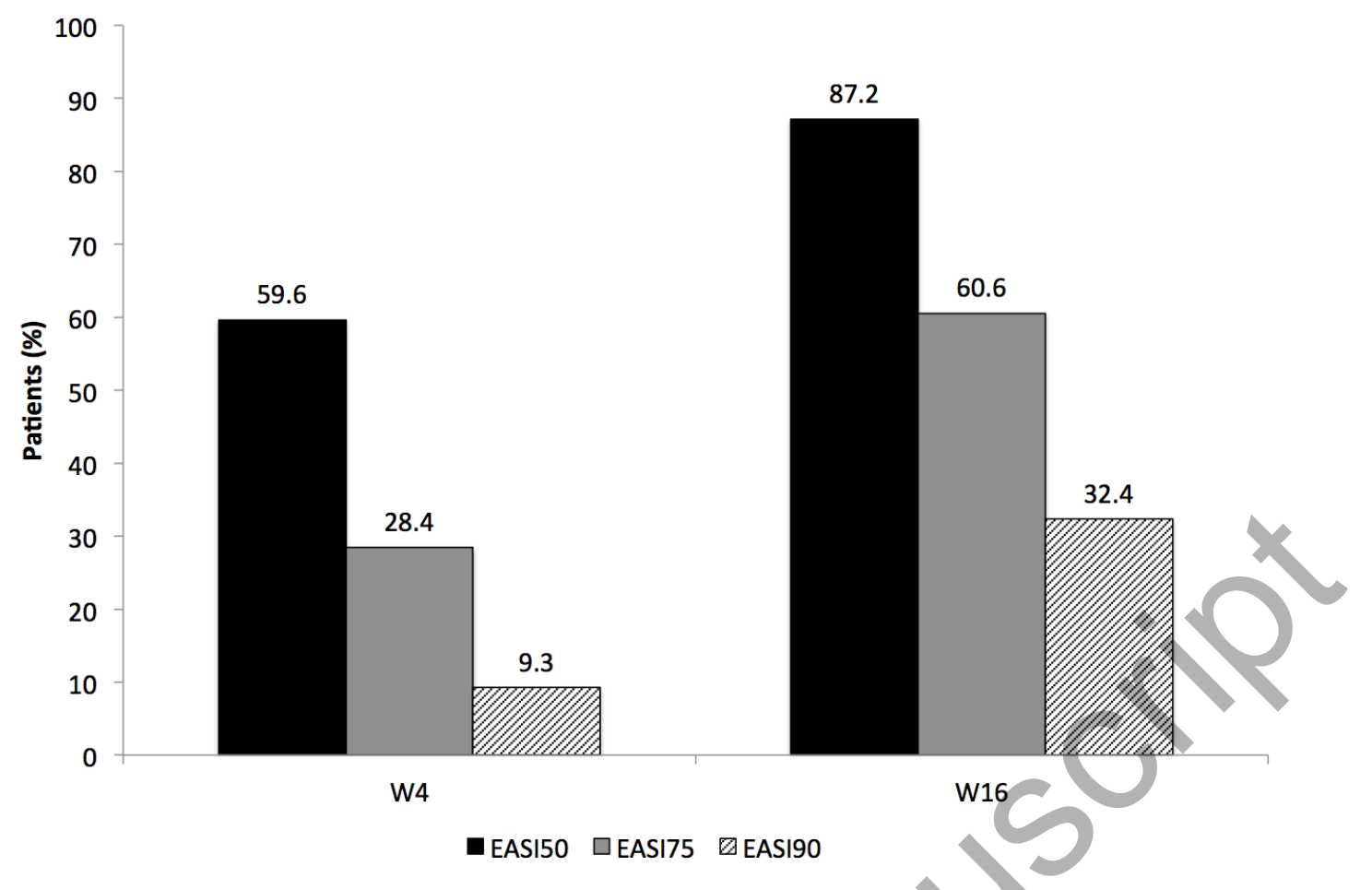

Figure 2. Percentage of patients achieving EASI50, EASI75 and EASI90 at week 4 (W4) and at week 16 (W16). Mean values are indicated on the bars. 

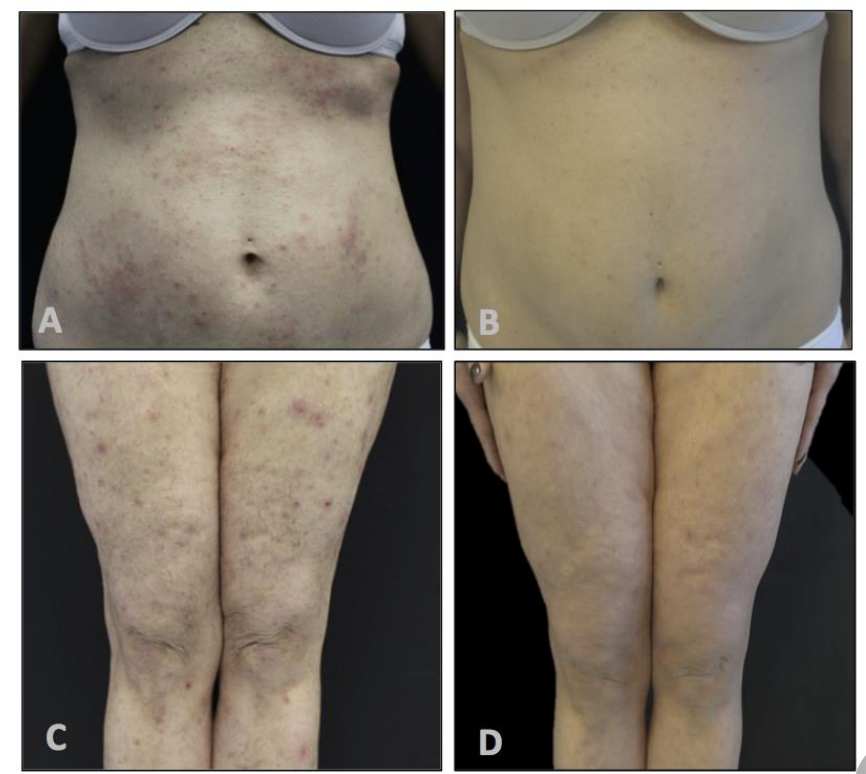

Figure 3. A 40 year-old patient affected by a persistent moderate-to-severe AD presenting with a classic adult-type phenotype before treatment (A,C - EASI score 24) and after 16 weeks of dupilumab treatment (B,D - EASI score 2). 